\title{
Mucocutaneous Leishmaniasis Mimicking Squamous Cell Carcinoma ${ }^{+}$
}

\author{
Rodolfo Mauceri 1,*, Giuseppina Campisi ${ }^{1,2}$, Claudia Colomba ${ }^{3}$, Olga Di Fede ${ }^{1}$ and \\ Vera Panzarella ${ }^{2}$ \\ 1 Department of Surgical, Oncological, and Oral Sciences, University of Palermo, 90127 Palermo, Italy; \\ campisi@odonto.unipa.it (G.C.); odifede@odonto.unipa.it (O.D.F.) \\ 2 Oral Medicine and Dentistry for patients with special needs, AUOP “P. Giaccone” of Palermo, \\ 90127 Palermo, Italy; panzarella@odonto.unipa.it \\ 3 Department of Sciences for Health Promotion and Mother-Child Care, University of Palermo, \\ 90127 Palermo, Italy; Claudia.colomba@unipa.it \\ * Correspondence: rodolfo.mauceri@unipa.it; Tel.: +39-09123864244 \\ + Presented at the XV National and III International Congress of the Italian Society of Oral Pathology and \\ Medicine (SIPMO), Bari, Italy, 17-19 October 2019.
}

Published: 10 December 2019

Leishmaniasis is a parasitic infectious disease caused by protozoan species belonging to the genus Leishmania. The leishmaniases are the third most important group of vectorborne diseases; it is endemic in 88 countries and it represents a major public health problem worldwide. In the Mediterranean area, most cases usually affect immunocompromised patients. Depending on the location of the lesions, the WHO described 11 human different clinical forms; the more common clinical forms can be categorized into four main groups: visceral leishmaniasis (VL); cutaneous leishmaniasis (CL); mucosal leishmaniasis (ML) and mucocutaneous leishmaniasis (MCL). Primary oral mucosal lesion can be the first sign of the infectious disease in all the clinical forms; usually oral lesions may be related to several symptoms (e.g., swallowing difficulties, dyspnea, dysphonia) [1-3]. In this case report, we presented a unusual case of MCL in a immunocompromised patients in South of Italy; whose presentation mimicked an oral squamous cell carcinoma (OSCC).

A Caucasian man was complaining about a cheeck lesion that began one-month prior the first visit appointment and was slowly involving the right lower lip. Patient reported to be affected by cutaneous psoriasis, in treatment with methotrexate and cyclosporine; additionally, he was a heavy smoker (Pack years: 26). Physical examination revealed a whitish, nodular and ulcerated lesion affecting the right oral commissure measuring approximately $2.5 \times 1.5 \mathrm{~cm}$, involving the lower lip right corner and buccal mucosa with hard consistency (Figure 1). Oral hygiene was poor, lower incisors were missing and mechanical trauma was present on the lesion. Palpation of the lesion revealed a firm consistency, the neck lymph nodes were slightly swelled.

Because a high suspicion of malignancy; the patient was included into a multidisciplinary care paths, named "GOTeC" ("Gruppo Oncologico Testa e Collo" - Policlinico "P. Giaccone" of Palermo), which deals with the entire diagnostic-therapeutic process of the patient suffering from H\&N cancers, from the suspected diagnosis to therapeutic complete plans. Patient underwent a magnetic resonance imaging that highlighted an oval lesion measuring $1.2 \times 2.9 \times 2.1 \mathrm{~cm}$ (Figure 2), The neoformation extends anteriorly to the outer profile of the lower lip involving the orbicular and contiguous buccinator muscles, there was the evidence of swollen submandibular lymph nodes. Subsequently, an incisional biopsy was carried out, which showed non-specific chronic inflammation without neoplastic signs. At the post-surgical follow-up visit, the patient reported the evolution of the cutaneous psoriatic-like cutaneous lesions on the right harm, knees and especially foot towards a suppurative aspect. The patient was then referred to the dermatologic and infectious disease unit for further investigations; he underwent abdominal ultrasound imaging, serological test and a 
polymerase chain reaction test on skin mucosa biopsy samples to investigate the main causes of skin and soft-tissue Infections. Both results showed the leishmania infection, so the diagnosis of mucocutaneous leishmaniasis was established. Patient was pharmacologically treatment with liposomal amphotericin B and topical therapy for psoriasis; after discontinuation of immunosuppressive therapy. At the latest follow-up visit at 8 months, a complete remission of the oral and skin lesions was observed.

As well as for OSCC, it is important to emphasizes the importance of a multidisciplinary approach in the diagnosis and treatment of mucocutaneous leishmaniasis, whose diagnosis remains a challenge due to the heterogeneous clinical presentation. The diagnostic difficulties shown in this case were related to the development of such lesion in a patient who lived in a non-endemic area and the high suspicious presentation of the oral lesion for OSCC. Additionally, even if the Leishmaniasis usually affects immunocompromised patients, such as transplant recipients or those who are affected by HIV, it should be evaluated also in patients with long-term immunosuppressive pharmacological treatments.

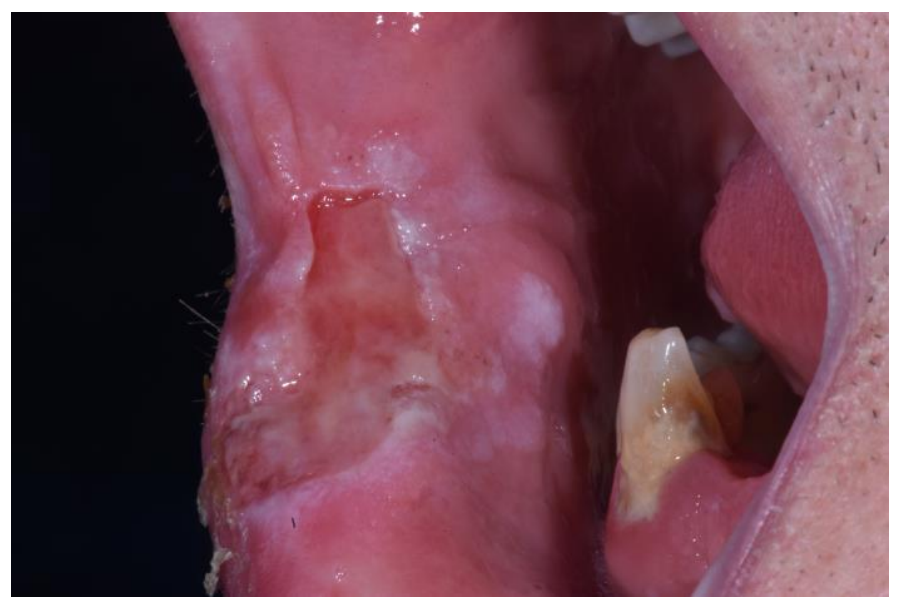

Figure 1. Clinical view of the oral lesion affecting the right labial commissure.

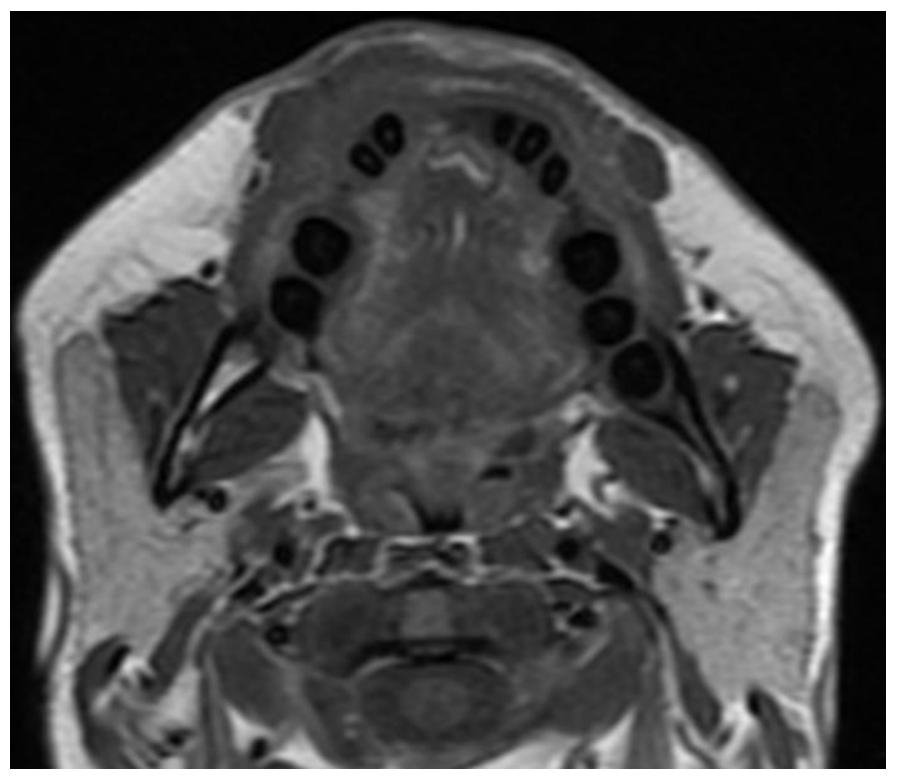

Figure 2. Magnetic resonance axial section.

Acknowledgments: This research received no external funding.

Conflicts of Interest: The authors declare no conflict of interest. 


\section{References}

1. Mignogna, M.D.; Celentano, A.; Leuci, S.; Cascone, M.; Adamo, D.; Ruoppo, E.; Favia, G. Mucosal leishmaniasis with primary oral involvement: A case series and a review of the literature. Oral Dis. 2015, 21, e70-e78.

2. Mohammadpour, I.; Motazedian, M.H.; Handjani, F.; Hatam, G.R. Lip leishmaniasis: A case series with molecular identification and literature review. BMC Infect. Dis. 2017, 17, 96.

3. Ramos, A.; Múñez, E.; García-Domínguez, J.; Martinez-Ruiz, R.; Chicharro, C.; Baños, I.; Suarez-Massa, D.; Cuervas-Mons, V. Mucosal leishmaniasis mimicking squamous cell carcinoma in a liver transplant recipient. Transpl. Infect. Dis. 2015, 17, 488-492.

(C) 2019 by the authors. Licensee MDPI, Basel, Switzerland. This article is an open access article distributed under the terms and conditions of the Creative Commons Attribution (CC BY) license (http://creativecommons.org/licenses/by/4.0/). 